\title{
Threshold Collision-Induced Dissociation of Hydrogen-Bonded \\ Dimers of Carboxylic Acids
}

Beike Jia, Laurence A. Angel, and Kent M. Ervin*

Department of Chemistry and Chemical Physics Program, University of Nevada, Reno, 1664 N. Virginia St. MS 216, Reno, NV 89557-0216

\section{SUPPORTING INFORMATION}

Geometries and molecular constants calculated at the B3LYP/aug-cc-pVDZ level using Gaussian $03,{ }^{1}$ except as noted. 
Table S1: Molecular parameters of ground-state product acids and bases ${ }^{a}$

\begin{tabular}{|c|c|c|}
\hline & $\mathrm{HNO}_{2}^{b}$ & $\mathbf{N O}_{2}^{-c}$ \\
\hline rotation $/ \mathrm{cm}^{-1}$ & $3.117,0.393(2)$ & $3.938,0.433(2)$ \\
\hline vibration $/ \mathrm{cm}^{-1}$ & $\begin{array}{l}549,607,795,1265,1684, \\
3552\end{array}$ & $776,1242,1284$ \\
\hline polarizability / $\mathrm{m}^{-30} 4 \pi \varepsilon_{0}$ & 3.11 & \\
\hline dipole moment / Debye & 2.05 & \\
\hline rotational symmetry & 1 & 2 \\
\hline$H_{298}-H_{0} / \mathrm{kJ} \mathrm{mol}^{-1}$ & $11.6 \pm 0.2^{b}$ & $10.21 \pm 0.12$ \\
\hline \multirow[t]{2}{*}{$S_{298}-S_{0} / \mathrm{J} \mathrm{mol}^{-1} \mathrm{~K}^{-1}$} & $254.1 \pm 0.7^{b}$ & $237.1 \pm 1.8$ \\
\hline & $\mathbf{H C O O H}^{d}$ & $\mathrm{HCOO}^{-}$ \\
\hline rotation $/ \mathrm{cm}^{-1}$ & $2.868,0.363(2)$ & $3.557,0.383(2)$ \\
\hline vibration $/ \mathrm{cm}^{-1}$ & $\begin{array}{l}625,638,1033,1105,1229 \\
1387,1770,2943,3570\end{array}$ & $\begin{array}{l}732,1030,1331,1355 \\
1648,2593\end{array}$ \\
\hline polarizability / $\mathrm{m}^{-30} 4 \pi \varepsilon_{0}$ & 3.40 & \\
\hline dipole moment / Debye & 1.41 & \\
\hline rotational symmetry & 1 & 2 \\
\hline$H_{298}-H_{0} / \mathrm{kJ} \mathrm{mol}^{-1}$ & $10.9 \pm 0.3$ & $10.32 \pm 0.16$ \\
\hline \multirow[t]{2}{*}{$S_{298}-S_{0} / \mathrm{J} \mathrm{mol}^{-1} \mathrm{~K}^{-1}$} & $249 \pm 3$ & $238.7 \pm 2.0$ \\
\hline & $\mathrm{CH}_{3} \mathrm{COOH}^{d}$ & $\mathrm{CH}_{3} \mathrm{COO}^{-}$ \\
\hline rotation $/ \mathrm{cm}^{-1}$ & $0.177,0.344(2)$ & $0.183,0.354(2)$ \\
\hline vibration $/ \mathrm{cm}^{-1}$ & $\begin{array}{l}\mathrm{HR}^{e}, 534,581,642,657,847, \\
989,1048,1182,1264,1382, \\
1430,1430,1788,2944,2996, \\
3051,3583\end{array}$ & $\begin{array}{l}\mathrm{FR},{ }^{f} 433,594,624,865, \\
984,1015,1295,1349, \\
1431,1443,1635,2998, \\
3064,3085\end{array}$ \\
\hline polarizability $/ \mathrm{m}^{-30} 4 \pi \varepsilon_{0}$ & 5.10 & \\
\hline dipole moment / Debye & 1.74 & \\
\hline rotational symmetry & $1[3]^{i}$ & $1[6]^{i}$ \\
\hline$H_{298}-H_{0} / \mathrm{kJ} \mathrm{mol}^{-1}$ & $13.6 \pm 0.7$ & $13.2 \pm 0.5$ \\
\hline \multirow[t]{2}{*}{$S_{298}-S_{0} / \mathrm{J} \mathrm{mol}^{-1} \mathrm{~K}^{-1}$} & $293 \pm 5$ & $272 \pm 5$ \\
\hline & $\mathrm{C}_{6} \mathrm{H}_{5} \mathrm{COOH}$ & $\mathrm{C}_{6} \mathrm{H}_{5} \mathrm{COO}^{-}$ \\
\hline rotation $/ \mathrm{cm}^{-1}$ & $0.129,0.036(2)$ & $0.130,0.036(2)$ \\
\hline vibration $/ \mathrm{cm}^{-1}$ & $\begin{array}{l}\mathrm{HR}^{g}{ }^{g} 163,216,382,417,443 \\
496,616,626,633,708,724\end{array}$ & $\begin{array}{l}\mathrm{HR}^{d}{ }^{d} 162,215,366,424, \\
456,500,626,672,706,\end{array}$ \\
\hline
\end{tabular}


$771,828,858,959,997,1009$, 1016, 1043, 1088, 1110, 1174, 1186, 1209, 1328, 1360, 1367, $1468,1512,1623,1645,1776$, 3175, 3187, 3197, 3211, 3218, 3755

polarizability / $\mathrm{m}^{-30} 4 \pi \varepsilon_{0}$

dipole moment / Debye rotational symmetry

$\mathrm{H}_{298}-\mathrm{H}_{0} / \mathrm{kJ} \mathrm{mol}^{-1}$

$S_{298}-S_{0} / \mathrm{J} \mathrm{mol}^{-1} \mathrm{~K}^{-1}$
13.56

2.18

$1[2]^{i}$

$21.2 \pm 1.7$

$354 \pm 11$
$712,806,829,852,938$ 992, 992, 1004,1035, 1065, $1118,1153,1165,1295$, $1338,1351,1449,1486$, $1606,1625,1660,3132$, 3143, 3163, 3190, 3191

${ }^{a}$ Calculated using the B3LYP/aug-cc-pVDZ method with Gaussian 03, except as noted. 2-D rotational constant is geometric mean of $A$ and $B$ or of $B$ and $C$.

${ }^{b}$ Vibrational frequencies are from reference 2. $H_{298}$ and $S_{298}$ including cis isomer taken from Gurvich et al. ${ }^{6}$

${ }^{c}$ References 3,4

${ }^{d}$ Reference 5 .

${ }^{e}$ Hindered methyl rotor, $V_{0}=131 \mathrm{~cm}^{-1}, B=5.6 \mathrm{~cm}^{-1}, n=3, \sigma^{\prime}=3$.

${ }^{f}$ Free methyl rotor, $B=5.6 \mathrm{~cm}^{-1}, \sigma^{\prime}=6$ (calculated barrier less than $2 \mathrm{~cm}^{-1}$ ).

${ }^{g}$ Hindered phenyl rotor, $V_{0}=2354 \mathrm{~cm}^{-1}, B=0.60 \mathrm{~cm}^{-1}, n=2, \sigma^{\prime}=2$.

${ }^{h}$ Hindered phenyl rotor, $V_{0}=1399 \mathrm{~cm}^{-1}, B=0.60 \mathrm{~cm}^{-1}, n=2, \sigma^{\prime}=4$.

${ }^{i}$ Symmetry number including internal rotors, in brackets.

\section{Table S2: Structures of Bases and Acid Isomers ${ }^{a}$}

B3LYP/aug-cc-pVDZ, Cartesian coordinates in Ångstroms.

\section{$\mathrm{CH}_{3} \mathrm{COO}^{-}$}

$C, 0,1.3523558526,-0.0303011816,-0.0073763036$

$C, 0,-0.2069808526,0.0038930881,-0.0087383308$

$0,0,-0.7294036159,1.1501737441,-0.0809450212$

$0,0,-0.7793094162,-1.1165306272,0.0871079537$

$\mathrm{H}, 0,1.7264028512,-0.9763862328,-0.4265721897$

$\mathrm{H}, 0,1.7659838556,0.8256022254,-0.5615084497$

$\mathrm{H}, 0,1.7050675496,0.0400876331,1.0354649863$

\section{$s-\mathrm{Z} \mathrm{CH} 3 \mathrm{COOH}$ ("cis")}

$\mathrm{C}, 0,0.6295662156,-1.1700612353,0.4451695929$

$\mathrm{H}, 0,0.6491090249,-1.1359186789,1.5428407151$

$\mathrm{H}, 0,1.6709743948,-1.1359180724,0.0977033685$

$\mathrm{H}, 0,0.1477729049,-2.0903389017,0.1044902388$

$\mathrm{C}, 0,-0.1246831455,0.0176634974,-0.0881641998$

$0,0,-1.1128141536,-0.0199314466,-0.7868776358$ 
$0,0,0.4382204127,1.1914763325,0.3098688078$

$\mathrm{H}, \Theta,-\Theta .1004048185,1.9042029939,-0.0709960562$

\author{
s- $\mathrm{E} \mathrm{CH}_{3} \mathrm{COOH}$ ("trans") \\ C $, 0,0.5988431168,-1.1656413664,0.423445105$ \\ $\mathrm{H}, 0,0.6127456753,-1.158530914,1.523286247$ \\ $\mathrm{H}, 0,1.6404171308,-1.1585303046,0.0699378439$ \\ $\mathrm{H}, 0,0.1037826092,-2.0759438171,0.073384441$ \\ $\mathrm{C}, 0,-0.149742809,0.0403588869,-0.10588403$ \\ $0,0,-1.1317663471,-0.0097597972,-0.8002788407$ \\ $0,0,0.3575134577,1.2586678249,0.2528004583$ \\ $\mathrm{H}, 0,1.1424758535,1.1534356913,0.8078520769$
}

\title{
$\mathrm{HCOO}^{-}$
}

$\mathrm{H}, 0,-0.1580563783,-0.0508031501,-1.4508634282$

$\mathrm{C}, 0,-0.0344855767,-0.0110848746,-0.3165694039$

$0,0,1.1561902582,0.0521703013,0.0843543132$

$0,0,-1.1105690284,-0.0375062516,0.3344306682$

\section{s-Z HCOOH ("cis")}

$\mathrm{H}, 0,-0.1570863731,-0.0522129293,-1.4987069974$

C $, \odot,-\odot .1488931553,-\odot .0183996,-\odot .3958568387$

$0,0,1.1228889038,0.0497093703,0.0503428121$

$0,0,-1.1279545532,-0.0391129423,0.3060636244$

$\mathrm{H}, 0,1.0909705006,0.0778391057,1.0225965379$

\section{$s-E$ HCOOH ("trans")}

$\mathrm{H}, 0,-0.2568964848,-0.0549525599,-1.4478414387$

C, $\odot,-\odot .184642403,-\odot .0182701759,-\odot .3410323264$

$0,0,1.0884725356,0.0503897375,0.1214365242$

$0,0,-1.1315608418,-0.0366298778,0.3930018072$

$\mathrm{H}, 0,1.7094573526,0.0544947377,-0.621471254$

$\mathrm{NO}_{2}^{-}$

$0,0,-0.3765256206,-0.0472189476,-1.0237886575$

$\mathrm{N}, 0,-0.3955055781,-0.0097878484,0.2367485591$

$0,0,0.7225930014,0.0557833149,0.8166336683$

s-cis HONO ("cis")

$0,0,-0.4069815765,-0.0481614005,-1.0117767352$

$\mathrm{N}, 0,-0.5222059753,-0.0173088251,0.1680431734$

$0,0,0.6901146154,0.0552555877,0.8451762329$

$\mathrm{H}, \Theta, 1.3903775163,0.064408278,0.1565018051$

\section{s-trans HONO ("trans")}

$0,0,-0.3726381601,-0.0482431281,-1.0630428997$

$\mathrm{N}, 0,-0.5100781332,-0.0188160985,0.1012211448$ 
$0,0,0.755486905,0.0555308905,0.7617935657$

$\mathrm{H}, 0,0.5077569733,0.0734105903,1.7014466581$

\section{$\mathrm{C}_{6} \mathrm{H}_{5} \mathrm{CO}_{2}^{-}$}

C, $0,-0.2301957513,0.0000000517,-1.2609887764$

$C, 0,-0.2434160299,-0.0000031215,0.1422356201$

$\mathrm{C}, 0,0.985480439,-0.0000038663,0.8196549119$

C, $0,2.1962027991,-0.00000013785,0.1172930778$

$C, 0,2.1970540378,0.0000017624,-1.2837704441$

$\mathrm{C}, 0,0.9760566601,0.0000002349,-1.970904246$

C, $\odot,-1.5831313612,-0.0000059502,0.9250184198$

$0,0,-1.4765397152,0.00000002829,2.1796706054$

$0,0,-2.623872189,0.0000072793,0.2163001952$

$\mathrm{H}, 0,0.9562637022,-0.00000067024,1.9096449221$

$\mathrm{H}, 0,3.1445681731,-0.0000018917,0.6612791896$

$\mathrm{H}, 0,3.1404914958,0.000003688,-1.8350445801$

$\mathrm{H}, 0,0.9677940788,0.0000046214,-3.0641900111$

$\mathrm{H}, 0,-1.1941269785,0.0000007071,-1.7706873042$

\section{s- $\mathrm{Z} \mathrm{C}_{6} \mathrm{H}_{5} \mathrm{COOH}$ ("cis")}

$\mathrm{C}, 0,-0.2025648506,-0.00000004148,-1.3148007467$

$C, 0,-\odot .2049150968,-\odot .0000050352,0.0893968876$

C, $0,1.0116048593,-0.0000046667,0.7910051225$

C, $0,2.2189899256,-0.00000002666,0.0896461536$

C, $0,2.2178462973,0.00000038375,-1.3094140784$

C, $0,1.0060300618,0.0000035819,-2.0105927425$

C, $0,-1.5187340152,-0.0000096858,0.7879612718$

$0,0,-1.4040666452,-0.0000025724,2.1443860139$

$0,0,-2.601380215,0.0000104265,0.2370018518$

$\mathrm{H}, 0,1.0080862726,-0.00000082175,1.8791409584$

$\mathrm{H}, 0,3.1630491574,-0.0000000557,0.6350944969$

$\mathrm{H}, 0,3.1627051607,0.0000071971,-1.8544293448$

$\mathrm{H}, 0,1.0055296156,0.0000065614,-3.100856186$

$\mathrm{H}, 0,-1.1555696728,-0.00000003755,-1.8416429754$

$H, \odot,-2.3097687401,0.0000079562,2.4923789184$

\section{s- $\mathrm{E} \mathrm{C}_{6} \mathrm{H}_{5} \mathrm{COOH}$ ("trans")}

$C, 0,0.9736177107,-0.1015475992,-2.0081005461$

C, $\odot,-\odot .2241176752,-0.0602710368,-1.2949930289$

C, $0,-0.2124932644,0.013466671,0.1074077383$

$\mathrm{C}, 0,1.0191091753,0.0639356737,0.7804811216$

C $, 0,2.2186389562,0.0329975862,0.0648037583$

C, $0,2.1972937486,-0.055191804,-1.3300975061$

C, $0,-1.5400549866,0.0577558189,0.8073384702$

$0,0,-2.579759503,0.3022864297,0.2428321989$

$0,0,-1.5521556175,-0.1965445054,2.1463382533$

$\mathrm{H}, 0,1.0673985423,0.1622171047,1.8667792109$ 
$\mathrm{H}, 0,3.1687108511,0.0835893112,0.5966578859$

$\mathrm{H}, 0,3.133524474,-0.0827835823,-1.8884332617$

$\mathrm{H}, 0,0.9544376206,-0.1666495269,-3.0961745395$

$H, 0,-1.184722155,-0.0842551587,-1.8068035335$

$\mathrm{H}, \Theta,-\odot .6759903559,-\odot .4649254019,2.4535705771$ 
Table S3: Structures and Frequencies of Complex Isomers

B3LYP/aug-cc-pVDZ, Cartesian coordinates in Ångstroms, vibrational frequencies in $\mathrm{cm}^{-1}$.

\section{(1) $\left[\mathrm{CH}_{3} \mathrm{COO} . . H . . O O C H\right]^{-}$}

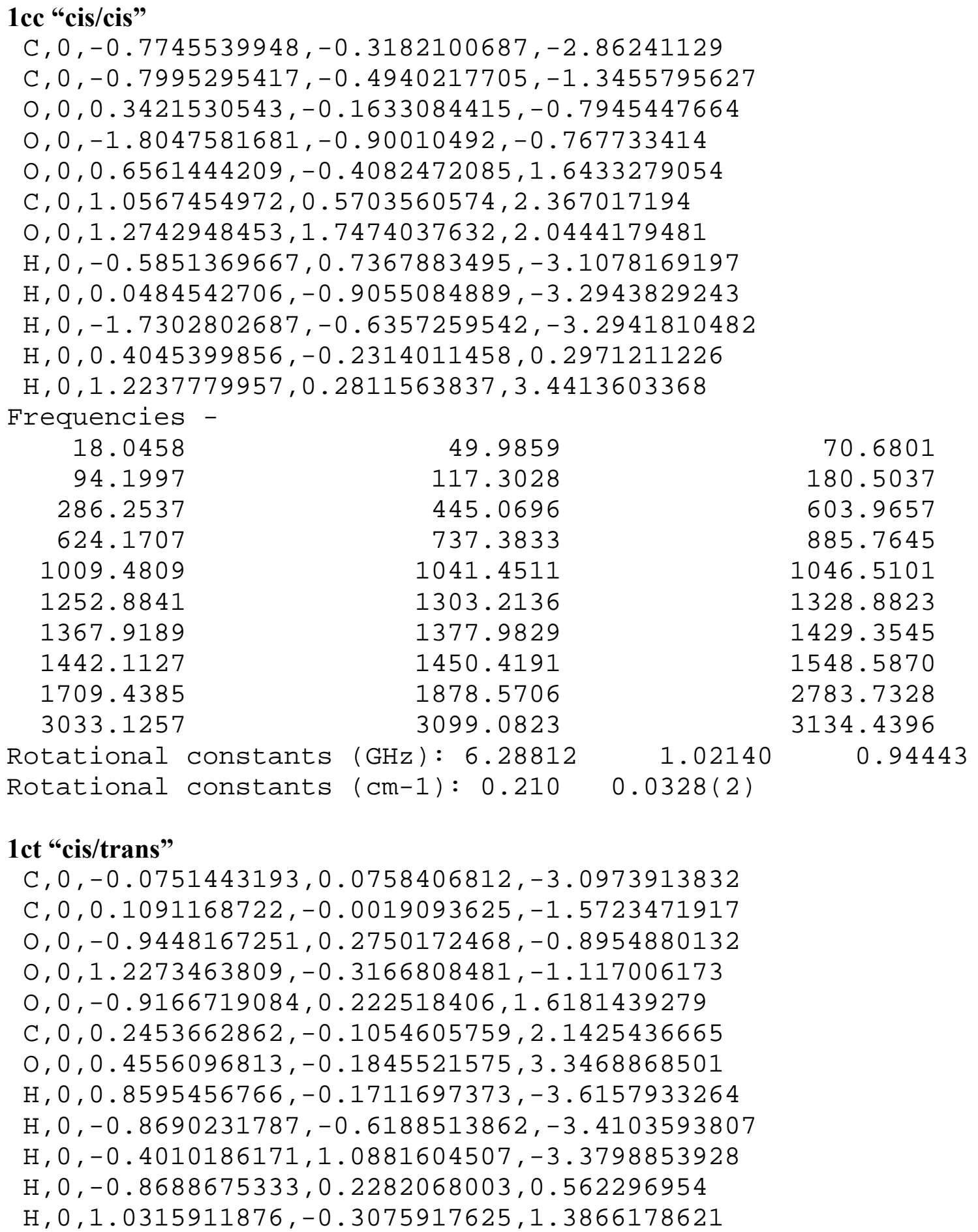


Frequencies -

36.9270

52.1941

73.1893

79.0064

156.9039

185.4012

249.3920

467.6451

610.2329

641.5985

698.1575

901.0463

1005.4489

1034.6728

1082.5419

1206.1169

1251.5110

1320.0016

1382.0863

1398.7328

1435.5367

1449.9767

1520.9541

1642.5403

1747.7266

3021.0322

2224.4390

3086.3563

2988.5188

3116.4027

Rotational constants (GHZ): 7.71602

Rotational constants $(\mathrm{cm}-1): 0.2570 .0322(2)$

1 te "trans/cis"

C, $0,0.777695061,-0.7291832439,-1.2274052359$

C, $0,0.9435352616,-1.3469429634,0.1500536917$

$0,0,1.7995420302,-2.1926562784,0.3886619833$

$0,0,0.1008579226,-0.9330799233,1.0850385946$

$0,0,-1.6592146476,0.7955216817,0.4632452518$

C, $0,-1.4325763366,1.9228091666,-0.088218397$

$0, \odot,-\odot .3649108345,2.3648013433,-\odot .5527368263$

$\mathrm{H}, 0,1.6079220613,-1.0466271109,-1.8679552267$

$\mathrm{H}, 0,-0.1732103756,-1.0702952057,-1.6637439321$

$\mathrm{H}, 0,0.7176949551,0.3670808252,-1.1593937462$

$H, 0,-0.5562486125,-0.1943668017,0.7656571116$

$\mathrm{H}, \Theta,-2.3382777096,2.5874159512,-0.1548165863$

Frequencies -

53.5917

132.2540

77.3048

171.0668

270.5851

460.0401

622.6871

741.3326

1020.1965

1164.4103

1047.3733

1374.8602

1307.9623

1457.0160

1386.1615

1746.0665

1480.7776

2483.3160

3021.2859

3089.8469

Rotational constants (GHZ): 5.48951

Rotational constants (GHZ): 0.183

1.25168

$0.0391(2)$

109.7878

181.8118

598.4260

891.5233

1052.6308

1343.6911

1444.5446

1650.5297

2781.3600

3135.9400

1 tt "trans/trans"

C $, 0,1.6346464395,0.2480000465,0.8959832206$

$0,0,0.4080899336,-\odot .1665197614,1.1422585925$

$0,0,2.5042176016,0.282959229,1.7625312963$

$\mathrm{C}, 0,1.9220736831,0.6865996004,-0.5348325466$

$\mathrm{H}, 0,2.9663141281,1.0071419444,-0.614177363$ 
$\mathrm{H}, 0,1.7197479961,-0.1376242073,-1.2323400171$ $\mathrm{H}, 0,1.2469893379,1.5034761315,-0.8242612122$ $\mathrm{H}, 0,-0.239421151,-0.140022225,0.2858028678$ $0,0,-1.0744538958,-0.0973265512,-0.8529585579$ C, $0,-2.2870413407,-0.4859215626,-0.6948925064$ $0,0,-3.1843879744,-0.5324382604,-1.5471214696$ $\mathrm{H}, 0,-2.5394283225,-0.8184373965,0.3497478287$ Frequencies -

$$
26.5123
$$

89.9461

273.9673

610.4188

1011.8881

1228.7523

1380.4411

1450.3753

1750.0142

3044.3790

Rotational constants (GHZ): 8.78176

Rotational constants (cm-1): 0.296

(2) $[\mathrm{HCOO} . . \mathrm{H} . . \mathrm{ONO}]^{-}$

2 cc "cis/cis"

C, $0,2.0768076902,0.3553629056,-0.0140012085$

$0,0,0.9193454599,0.7915956327,-0.4373348351$

$0,0,2.3556177328,-0.7316047799,0.4710884032$

$\mathrm{H}, 0,2.861732754,1.1362955426,-0.147091481$

$\mathrm{H}, 0,0.0889235413,0.1128028961,-0.3449749066$

$0,0,-1.0370525965,-0.7988802565,-0.4490047313$

$\mathrm{N}, 0,-2.1928421738,-0.4293890923,-0.0193555728$

$0,0,-2.2456114988,0.6919453754,0.5041964943$

Frequencies -

$$
12.4859
$$

169.7610

710.1552

1170.2093

1383.7842

1692.8691

Rotational constants (GHZ): 9.04513

Rotational constants (cm-1): 0.302

101.4244

215.1713

798.3734

1267.4298

1435.3513

2010.5412

1.48006

$\odot .0475(2)$

\section{2ct "cis/trans"}

$\mathrm{H}, 0,3.0813077893,1.0731885887,-0.0016260507$

C, $0,2.2518304718,0.3163922597,-0.0007502393$

$0,0,2.5513961661,-0.883028934,0.0002933837$

$0,0,1.086038018,0.8595142546,-\odot .0 \odot 10019691$ 
$\mathrm{H}, 0,0.0038552553,0.0896756075,-\odot .0000912799$

$0, \odot,-\odot .9547572789,-0.5044800071,0.0006287251$

$\mathrm{N}, 0,-1.9516880414,0.3581874058,0.0000912454$

$0,0,-3.0494681033,-0.168071513,0.0007773664$

Frequencies -

14.1333

154.3888

708.2942

1048.0474

1352.9299

1669.0496
83.0035

195.6363

747.6145

1235.5086

1393.8241

1760.8599
130.2529

338.7752

1028.3826

1315.9137

1545.2308

2810.2747

Rotational constants (GHZ): $\begin{array}{llll}15.34383 & 1.23267 & 1.14100\end{array}$

Rotational constants (cm-1): $0.0512 \quad 0.0396(2)$

2 tc "trans/cis"

$\mathrm{C}, 0,0.579316148,-0.2190409636,-1.5027066409$

$\mathrm{H}, 0,0.9663018839,0.5810664076,-0.8410555293$

$0, \odot,-\odot .371665438,-\odot .9452142633,-\odot .9465663146$

$0,0,-1.0491894436,-0.3188945004,1.4626579402$

$\mathrm{N}, 0,-0.5067276075,0.6432280148,2.1091540808$

$0,0,0.380735035,1.2723217353,1.5043087758$

$0,0,1.0000536263,-0.4019224124,-2.635947788$

$\mathrm{H}, 0,-0.574575758,-0.6197412029,0.0175959018$

Frequencies -

$$
46.1963
$$

162.0443

700.9550

1143.1286

1386.1211

1757.2757

Rotational constants ( $\mathrm{GHZ})$ :

Rotational constants (GHZ):
102.5124

206.0100

809.7900

1219.8149

1402.9099

2518.2830
132.5839

265.2191

1075.6428

1253.4348

1524.1721

3001.5571

1.31440

\section{2tt "trans/trans"}

$\mathrm{C}, 0,1.7832440977,-0.322585027,0.0007281308$

$\mathrm{H}, 0,1.048502192,-1.1559154026,0.0017604628$

$0,0,1.2331270137,0.870053376,-0.001005087$

$0,0,-1.2762822374,0.8167769256,-0.0013457573$

$\mathrm{N}, 0,-1.7105414793,-0.3995748048,0.0002920578$

$0,0,-2.9382102055,-0.5198491438,0.0002557342$

$0,0,2.98834362,-0.532024179,0.0012097209$

$\mathrm{H}, 0,0.1699980497,0.8087933677,-0.0010905392$

Frequencies -

15.8158

167.9130

697.4494

1173.5908
73.8950

188.5463

821.2617

1214.4680
117.4826

277.5569

1061.3457

1265.6285 
1398.3017

1757.3006

Rotational

Rotational constants $(\mathrm{cm}-1)$ :

\section{(3) [CH3COO..H..ONO $]^{-}$}

1588.9871

2961.4934

1461.3001

1.12639

$13.61674 \quad 1.22797$

$0.45420 .0392(2)$

\footnotetext{
3ct "cis/trans"

$\mathrm{C}, 0,-3.0149980717,-0.7222988099,-0.0000129091$

$\mathrm{C}, 0,-1.7507499867,0.1340680647,-0.0000059091$

$0,0,-0.6610060589,-\odot .5932490435,0.0000510909$

$0,0,-1.8117258649,1.3612560707,-0.0000499091$

$0,0,1.5462900505,0.5086737374,0.0001100909$

$\mathrm{N}, 0,2.4158439553,-0.4499123489,-0.0000719091$

$0,0,3.5926579918,-0.0823494657,-0.0000369091$

$\mathrm{H}, 0,-3.9040170081,-0.0819097216,-0.0000599091$

$\mathrm{H}, 0,-3.0222841365,-1.3749558091,-0.8848789091$

$\mathrm{H}, 0,-3.0223341365,-1.3748968091,0.8848960909$

$\mathrm{H}, 0,0.3024869956,-0.0441171391,0.0000640909$

Frequencies -
}

\author{
72.3072 \\ 184.9470 \\ 601.8476 \\ 888.2064 \\ 1174.6010 \\ 1361.7361 \\ 1450.1944 \\ 2190.4996 \\ 3138.2762 \\ 0.96251
}




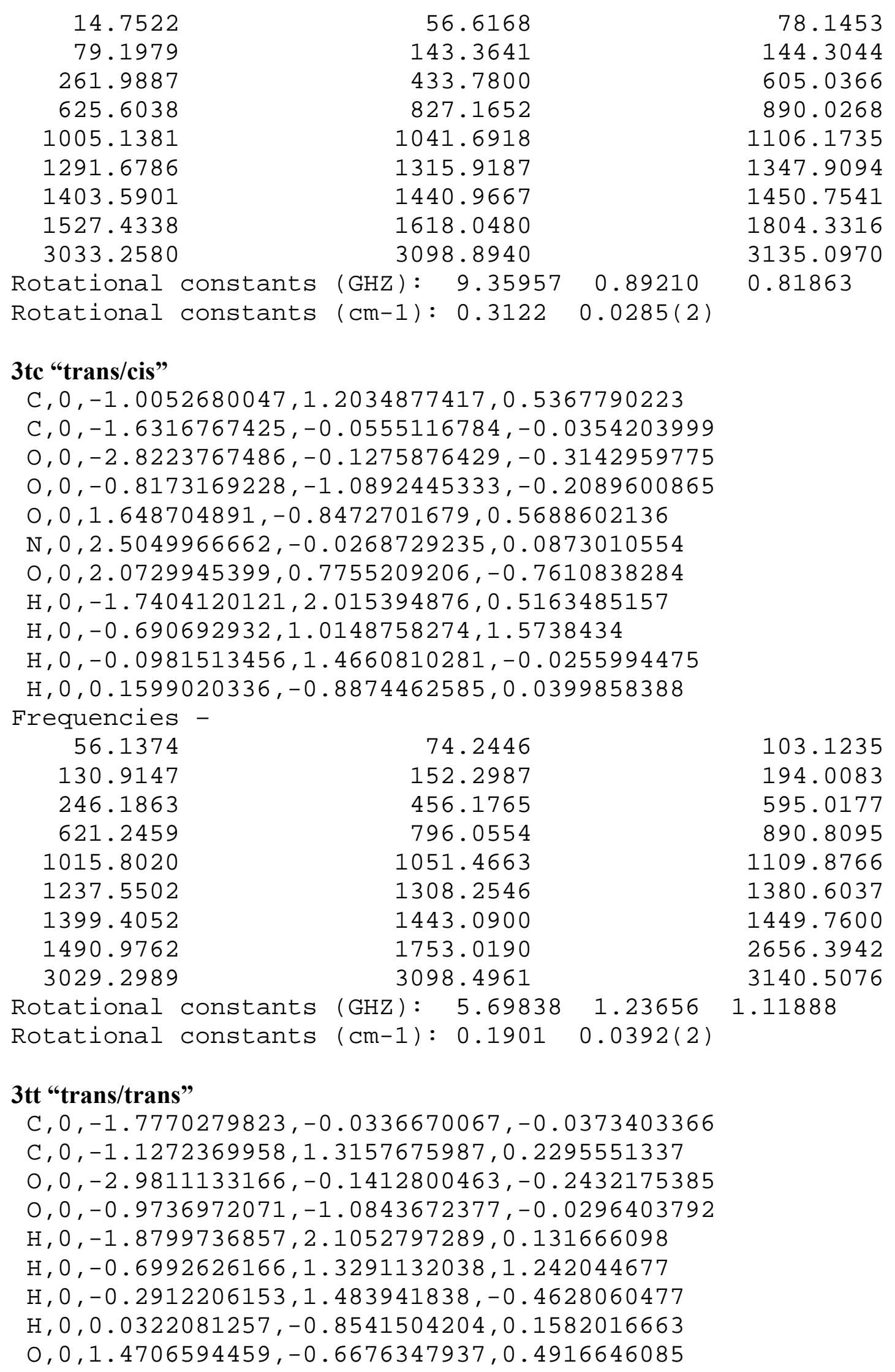


$\mathrm{N}, 0,2.0296936341,0.1306058431,-0.3517519484$

$0,0,3.2411489805,0.3094034772,-0.188823133$

Frequencies -

$\begin{array}{rr}53.1714 & 68.3506 \\ 136.3616 & 141.0955 \\ 253.4993 & 458.1222 \\ 617.9556 & 817.2242 \\ 1013.5979 & 1047.2098 \\ 1232.6455 & 1314.5480 \\ 1437.1454 & 1449.8656 \\ 1516.4050 & 1748.3425 \\ 3039.0245 & 3107.1194\end{array}$

Rotational constants (GHZ): $7.50074 \quad 1.04319$

Rotational constants (cm-1): $0.25020 .0332(2)$
81.3045

161.5037

596.8632

888.5264

1179.9678

1379.8169

1461.1707

2324.9108

3144.0640

$\odot .94868$

\section{(4) $[\mathrm{C6H5O..H..OOCH}]^{-}$}
4cc "cis/cis"
C, $0,1.4594271771,1.1530138556,-0.0123872299$
C, $0,2.7548846565,1.6816679953,-0.0152097719$
$\mathrm{C}, 0,3.8639273691,0.8272160342,0.0038452867$
C, $0,3.6671691109,-0.5593017617,0.0257327272$
C, $0,2.3708072525,-1.0834487831,0.0284777629$
$\mathrm{C}, 0,1.2545670367,-0.2346499454,0.0094822875$
$\mathrm{C}, 0,-0.1572157922,-0.828963275,0.0128577701$
$0,0,-1.0963852296,0.0460376007,-0.0057816454$
$0,0,-0.2702280154,-2.0661809133,0.0326859265$
$0,0,-3.4680821808,-0.6563793294,-0.0050258097$
$\mathrm{C}, 0,-4.3678753637,0.2882143574,-0.0243692475$
$0,0,-4.2073185759,1.5018685005,-0.0433037816$
$\mathrm{H}, 0,0.5835408814,1.8003395858,-0.026984254$
$\mathrm{H}, 0,2.9014307171,2.7639165186,-0.0323250141$
$\mathrm{H}, 0,4.8754762421,1.2390609424,0.001657138$
$\mathrm{H}, 0,4.5281464051,-1.2314507802,0.0406706952$
$\mathrm{H}, 0,2.190760614,-2.1581234335,0.0453186709$
$\mathrm{H}, 0,-2.4211219183,-0.3347407717,-0.005524187$
$\mathrm{H}, 0,-5.3962696093,-0.144259793,-0.0219880778$
Frequencies -
11.2841
67.7823
184.3510
410.5217
546.3921
713.0180
823.4935
948.1398
38.3508
114.3133
210.1590
424.8004
626.7454
714.1697
836.1708
998.0129

43.5479

169.1388

279.8499

460.7365

673.4735

721.7025

856.9247

1002.4722 


$$
\begin{aligned}
& 1007.2476 \\
& 1037.8577 \\
& 1075.6361 \\
& 1134.5131 \\
& 1171.2574 \\
& 1277.9756 \\
& 1306.7835 \\
& 1385.8582 \\
& 1455.6613 \\
& 1495.9356 \\
& 1634.5232 \\
& 1550.3918 \\
& 2898.7335 \\
& 3170.7935 \\
& 1708.9746 \\
& 3142.4016 \\
& 3195.7266 \\
& \text { Rotational constants (GHZ): } 2.34001 \quad 0.37106 \\
& \text { Rotational constants (cm-1): } 0.78060 .0115(2)
\end{aligned}
$$

\section{4ct "cis/trans"}

Frequencies -

26.4612

80.5394

194.7457

405.1566

529.2856

701.1115

825.2677

948.0356

1007.4188

1080.4138

1169.0007

1305.6593

1400.5637

1516.5779

48.5576
125.2652
195.2807
424.6728
626.7516
713.0998
835.7751
998.0752
1038.2050
1134.6451
1173.1401
1351.8574
1455.3660
1610.1435

52.3328

164.1682

259.4696

460.7029

679.0618

713.6655

856.9697

1001.7519

1075.4505

1158.4249

1242.0355

1369.6528

1495.8018

1634.7553 
1654.2262

2993.8944

3171.7166

Rotational

Rotational

4tc "trans/cis"

C, $0,3.0476657239,-0.418810874,0.3594501051$

C $, 0,2.1869134795,0.6742485529,0.468953952$

C, $0,0.8523868108,0.5804119555,0.039033481$

$C, \odot, 0.3934668933,-0.6197346273,-0.5244442078$

C, $0,1.2659850325,-1.7078030964,-0.6498919383$

$C, 0,2.5880073866,-1.6159652713,-0.2054258337$

C, $0,-0.0154570563,1.8160853896,0.1557020373$

$0,0,0.5135736474,2.9249273131,0.1896386321$

$0,0,-1.3231201727,1.6743240096,0.1974664478$

$0,0,-2.5718239081,-0.3865625156,0.8646601923$

C $, 0,-2.999333983,-1.309018441,0.0934763474$

$0,0,-2.6888172495,-1.5352868836,-1.0907703645$

$\mathrm{H}, \Theta,-0.6364228551,-0.7246200782,-0.8709568986$

$\mathrm{H}, \Theta, 0.8979895665,-2.6339079013,-1.0941734172$

$H, 0,3.260262818,-2.4716451492,-\odot .2985766848$

$\mathrm{H}, 0,4.0791333689,-0.337919307,0.7084835214$

$\mathrm{H}, 0,2.5312979775,1.621682178,0.8818128005$

$\mathrm{H}, 0,-1.7423414673,0.7258551996,0.3884041698$

$\mathrm{H}, 0,-3.7462236706,-1.9951418593,0.5759235894$

Frequencies -

44.2911

94.7988

184.7682

380.8417

537.6053

705.5366

801.0170

950.6737

1027.4971

1095.6474

1179.6302

1333.2713

1373.0813

1511.4438

1648.5595

2806.1146

3166.1955

Rotational

Rotational

-

50.6806

117.1321

218.1893

418.7917

624.9435

717.0986

818.7060

985.6595

1042.2210

1137.3856

1205.7514

1341.1085

1386.6935

1617.9564

1713.6754

3147.9219

3181.1715

constants (GHZ): 1.18271

constants $(\mathrm{cm}-1): 0.02945$
2446.0092

3155.5540

3202.4705

0.30661
83.2014

163.6334

284.5004

431.3727

661.1875

738.3341

860.6944

1004.9625

1048.4679

1162.1581

1321.3951

1358.9922

1457.9507

1638.5912

2268.2532

3160.0302

3199.5700

$0.68533 \quad 0.46343$

$0.0188(2)$ 


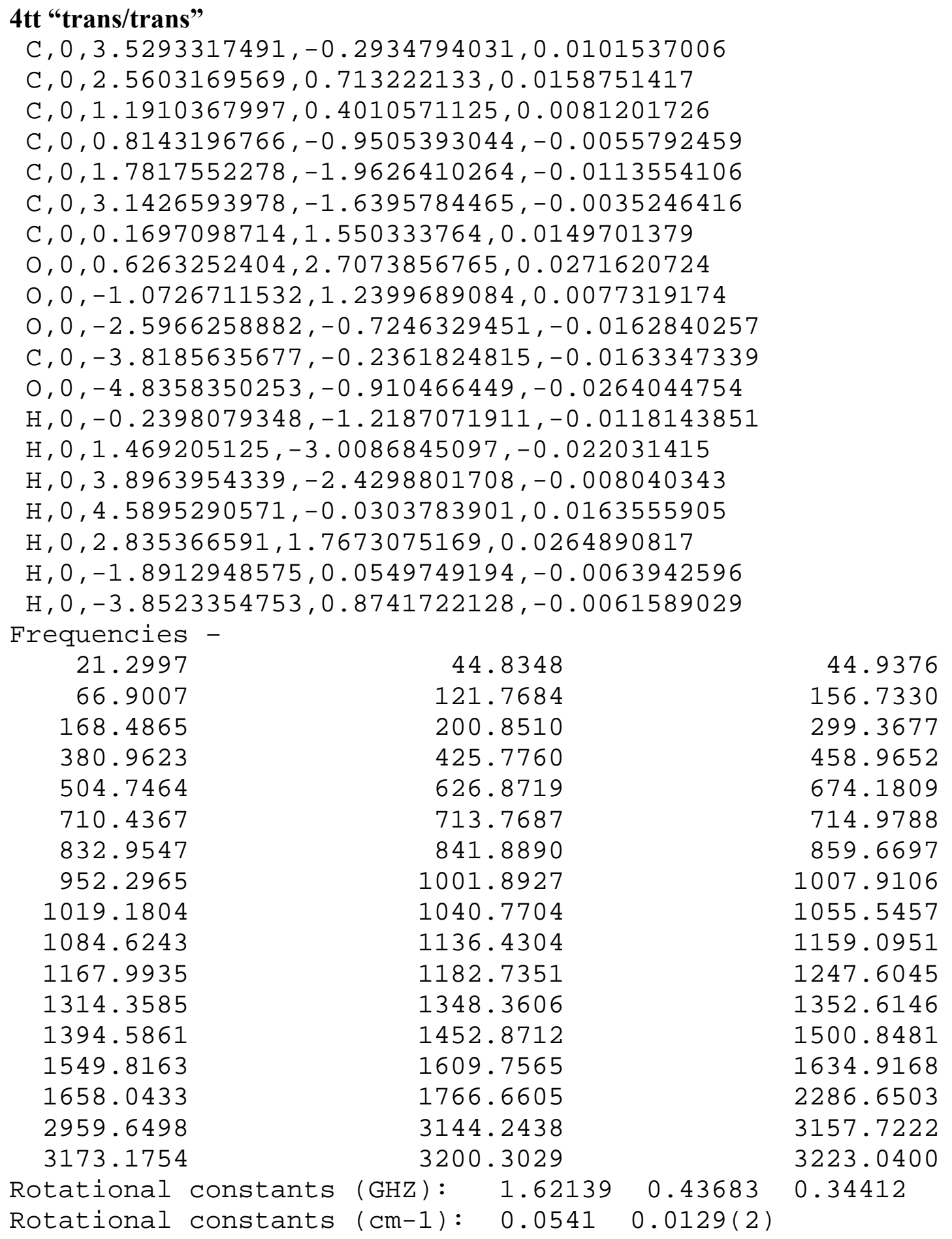

\section{(5) $\left[\mathrm{C}_{6} \mathrm{H}_{5} \text { O..H..ONO }\right]^{-}$}

\section{5 cc "cis/cis"}

C, $0,-1.4513576471,-1.1609887073,-0.0139263469$

C, $0,-2.7580335523,-1.6597675585,-0.0176727757$ 
$C, 0,-3.8471507032,-0.7809459123,0.0000053887$ $\mathrm{C}, 0,-3.6226702184,0.6011001092,0.0214766237$ C, $0,-2.3167657573,1.0979471502,0.0251912347$ C, $0,-1.2213412997,0.2227580904,0.0075529311$ $\mathrm{C}, 0,0.1838702611,0.7983436331,0.0122055227$ $0,0,1.1098667198,-0.1262359191,-0.0056750817$ $0,0,0.3545793942,2.0145864355,0.0311904573$ $0,0,3.488602779,0.6523764295,-0.0010549663$ $\mathrm{N}, 0,4.4246124001,-0.2352729177,-0.0182295636$ $0,0,4.0540946521,-1.4155143087,-0.0357102019$ $H, 0,-0.594271227,-1.8320769167,-0.027505466$ $H, 0,-2.9275226171,-2.7380425496,-0.034442409$ $\mathrm{H}, 0,-4.86688417,-1.1710821735,-0.0029307878$ $\mathrm{H}, 0,-4.4687230971,1.2909950175,0.0353243843$ $\mathrm{H}, 0,-2.1164721723,2.1687580212,0.0417373815$ $\mathrm{H}, 0,2.1451316241,0.215977099,-\odot .003573287$ Frequencies 13.7303 67.4124 192.8759 392.8903 506.2894 712.7514

827.7201 952.3917 1008.2785 1133.0096 1179.8416 1336.9762 1459.4825 1617.9496 1863.7500 3177.5074

43.4543 118.0549 197.2426 423.0639 627.0332 714.4753 834.5308 998.8529 1040.5239 1161.6303 1257.2876 1354.5909 1484.0050 1639.5655 3150.6105 3199.8778
51.9997 163.4824 278.7405 460.6571 671.3672 784.1689 858.4889 1005.2368 1082.6470 1162.1864 1312.4096 1421.9547 1504.8353 1648.4508 3163.7065 3211.1935 0.32476

Rotational constants (GHZ): $\quad 2.43900 \quad 0.37464$ Rotational constants (cm-1): $0.081400116(2)$

\section{5ct "cis/trans"}

C, $0,-3.7305911582,-0.5707662845,0.0984074873$

$\mathrm{C}, 0,-2.6254542186,0.2836927084,0.1573574988$

C, $0,-1.3226505653,-0.220291422,0.0325828066$

C $, 0,-1.1452792474,-1.5993907444,-0.1528625566$

C $, 0,-2.2482941671,-2.4580485588,-0.2124498841$

$\mathrm{C}, 0,-3.5455075477,-1.9465904525,-0.0869719357$

C, $0,-0.1273552254,0.7335329629,0.1001545576$

$0,0,-0.3518107035,1.9435174349,0.2660288444$

$0,0,1.0160311415,0.1609676905,-0.0260952608$

$0,0,3.1223410079,1.4583497366,0.0437009635$ 
$\mathrm{N}, 0,4.0801811476,0.5565041209,-0.1163367867$

$0,0,5.195111006,1.035379139,-0.1061126251$

$\mathrm{H}, 0,-0.1285803604,-1.978135033,-0.2483641625$

$\mathrm{H}, 0,-2.0971015841,-3.5299985435,-0.3572068075$

$\mathrm{H}, 0,-4.4071993415,-2.6159359107,-0.1333359882$

$\mathrm{H}, 0,-4.740028654,-0.1653649622,0.1970011339$

$\mathrm{H}, 0,-2.7428348736,1.3575526357,0.3008032564$

$\mathrm{H}, 0,2.1718899424,0.917811706,0.0179768554$

Frequencies -

$17.4594 \quad 41.8803$

67.9805

133.5526

183.8350

185.3199

411.1002

424.3278

545.0749

626.7960

712.7829

713.6253

822.9524

835.5772

947.9091

997.2609

1007.2869

1037.4673

1076.2731

1136.0800

1173.5403

1285.3078

1331.6224

1352.9153

1484.2561

1496.6976

1622.4581

1635.2227

1853.1648

3143.9335

3172 . 0201

3196.9182

Rotational constants (GHZ): 2.792230 .33232

Rotational constants (cm-1): $0.09310 .0105(2)$

42.1469

154.6551

281.3445

460.4216

679.2438

759.7601

856.7510

1001.2276

1062.5511

1158.6343

1305.8888

1455.9616

1590.7639

1686.1547

3156.0977

3204.1970

$\odot .29698$

\section{5 tc "trans/cis"}

C, $0,3.0383044491,-0.6351356203,-0.2266781455$

C , $0,2.2778537954,0.5299724939,-0.1195695529$

C, $0,0.9018144418,0.4622307073,0.1564546598$

C, $0,0.2975995452,-0.7896398739,0.3451122856$

C, $0,1.0676971574,-1.9551242694,0.2538734695$

C, $0,2.4330394883,-1.8845072218,-0.0367247947$

C, $0,0.1452915037,1.7656504065,0.28955286$

$0,0,0.7518207665,2.7992938492,0.5523954185$

$0,0,-1.1667747495,1.7617868424,0.137583929$

$0,0,-2.5048731902,-0.0053672429,-1.1513382445$

$\mathrm{N}, 0,-3.167614173,-0.9783269097,-0.6505465844$

$0,0,-2.9376524445,-1.2297475729,0.5479763384$

$\mathrm{H}, 0,-0.7689701343,-0.8661144531,0.5656803319$

$\mathrm{H}, 0,0.5881291687,-2.9232012922,0.4063827053$

$\mathrm{H}, 0,3.0260943132,-2.79812635,-0.1147920927$

$\mathrm{H}, 0,4.1043590816,-0.5715266168,-0.4535059911$

$\mathrm{H}, 0,2.7329215302,1.5121205335,-0.2414073959$

$\mathrm{H}, 0,-1.6190000923,0.9267298048,-0.2735956889$ 


$\begin{array}{rrr}\text { Frequencies } & & \\ 43.6816 & 52.4491 & 81.3281 \\ 94.3578 & 121.7315 & 165.0976 \\ 199.7375 & 215.7939 & 266.9293 \\ 381.4887 & 418.6103 & 433.4041 \\ 533.4353 & 625.4404 & 663.9069 \\ 706.0622 & 716.0095 & 790.2173 \\ 805.4369 & 818.8343 & 860.8246 \\ 952.4533 & 988.1380 & 1005.7517 \\ 1024.4261 & 1042.8609 & 1088.3953 \\ 1105.2088 & 1137.3776 & 1163.2862 \\ 1199.4848 & 1242.9431 & 1329.2053 \\ 1334.6564 & 1359.0030 & 1378.9829 \\ 1423.8974 & 1459.8114 & 1512.5275 \\ 1618.4672 & 1641.2591 & 1721.5550 \\ 2557.3812 & 3149.4223 & 3155.1830 \\ 3167.5026 & 3181.7672 & \\ \text { Rotational constants }(\mathrm{GHZ}): & 1.21400 & 0.66812 \\ \text { Rotational constants }(\mathrm{cm}-1): 0.460 .0456\end{array}$

\section{5tt "trans/trans"}

C, $0,-2.389782023,0.410186,0.372916$

C, $0,-1.064521023,0.427168,-0.090449$

$\mathrm{C}, 0,-0.476763023,-0.781533,-0.491693$

C, $0,-1.197598023,-1.979926,-0.430962$

C, $\odot,-2.512736023,-1.988634,0.045552$

C, $0,-3.106873023,-0.786456,0.451007$

$\mathrm{C}, 0,-0.317539023,1.762613,-0.17276$

$0,0,0.961643977,1.716744,-0.287613$

$0,0,-1.003646023,2.797761,-0.144737$

$0,0,2.702929977,0.265082,0.661699$

$\mathrm{N}, 0,3.066166977,-0.667696,-0.210726$

$0,0,3.998200977,-1.340772,0.168904$

$\mathrm{H}, \Theta, 0.547988977,-0.780872,-0.859354$

$\mathrm{H}, 0,-0.726944023,-2.911016,-0.752931$

$\mathrm{H}, 0,-3.072173023,-2.924973,0.10002$

$\mathrm{H}, 0,-4.133723023,-0.783972,0.823567$

$\mathrm{H}, 0,-2.839462023,1.35998,0.660806$

$H, 0,1.882985977,0.823697,0.215284$

Frequencies -

30.0046

83.6743

170.6393

378.8946

522.9768

707.3216

823.6821
42.4716

138.5396

208.0314

420.5169

626.1482

712.2650

844.0490
48.8717

157.2536

290.0341

435.9831

678.8610

755.8911

854.6739 


\begin{tabular}{|c|c|c|c|}
\hline 941.2425 & 988.5330 & & 990.8584 \\
\hline 1005.9832 & 1038.4835 & & 1055.6897 \\
\hline 1084.4463 & 1139.6260 & & 1159.6341 \\
\hline 1180.2897 & 1297.3827 & & 1308.3676 \\
\hline 1315.7731 & 1351.7520 & & 1454.3853 \\
\hline 1455.0581 & 1500.7119 & & 1610.4152 \\
\hline 1634.6123 & 1644.3302 & & 1655.2772 \\
\hline 1877.2735 & 3144.9009 & & 3158.7055 \\
\hline 3173.6130 & 3198.3193 & & 3207.8868 \\
\hline ational & 1.25832 & 0.56717 & 0.40467 \\
\hline tational constants & $(\mathrm{cm}-1): 0.0420$ & $0.0160(2)$ & \\
\hline
\end{tabular}




\section{References and Notes}

(1) Frisch, M. J.; Trucks, G. W.; Schlegel, H. B.; Scuseria, G. E.; Robb, M. A.; Cheeseman, J. R.; Montgomery, J. A., Jr.; Vreven, T.; Kudin, K. N.; Burant, J. C.; Millam, J. M.; Iyengar, S. S.; Tomasi, J.; Barone, V.; Mennucci, B.; Cossi, M.; Scalmani, G.; Rega, N.; Petersson, G. A.; Nakatsuji, H.; Hada, M.; Ehara, M.; Toyota, K.; Fukuda, R.; Hasegawa, J.; Ishida, M.; Nakajima, T.; Honda, Y.; Kitao, O.; Nakai, H.; Klene, M.; Li, X.; Knox, J. E.; Hratchian, H. P.; Cross, J. B.; Adamo, C.; Jaramillo, J.; Gomperts, R.; Stratmann, R. E.; Yazyev, O.; Austin, A. J.; Cammi, R.; Pomelli, C.; Ochterski, J. W.; Ayala, P. Y.; Morokuma, K.; Voth, G. A.; Salvador, P.; Dannenberg, J. J.; Zakrzewski, V. G.; Dapprich, S.; Daniels, A. D.; Strain, M. C.; Farkas, O.; Malick, D. K.; Rabuck, A. D.; Raghavachari, K.; Foresman, J. B.; Ortiz, J. V.; Cui, Q.; Baboul, A. G.; Clifford, S.; Cioslowski, J.; Stefanov, B. B.; Liu, G.; Liashenko, A.; Piskorz, P.; Komaromi, I.; Martin, R. L.; Fox, D. J.; Keith, T.; Al-Laham, M. A.; Peng, C. Y.; Nanayakkara, A.; Challacombe, M.; Gill, P. M. W.; Johnson, B.; Chen, W.; Wong, M. W.; Gonzalez, C.; Pople, J. A. Gaussian 03, Revision C.02, Pittsburgh, PA: Gaussian, Inc., 2004.

113.

(2) Khriachtchev, L.; Lundell, J.; Isoniemi, E.; Rasanen, M. J. Chem. Phys. 2000,

(3) Ervin, K. M.; Ho, J.; Lineberger, W. C. J. Phys. Chem. 1988, 92, 5405.

(4) Jacox, M.; Thompson, W. J. Chem. Phys. 1990, 99, 7393.

(5) Shimanouchi, T. Tables of Molecular Vibrational Frequencies Consolidated Volume I;. National Standard Reference Data System, 1972.

(6) Gurvich, L. V.; Veyts, I. V.; Alcock, C. B. Thermodynamic Properties of Individual Substances. $4^{\text {th }}$ ed., Vol. 1. Part 2. Hemisphere Publishing, New York, 1989.

Figures S1-S5. Schematic energy level diagrams showing conformers of the protonbound cluster ions and of the product acids. Dashed lines denote "diabatic" pathways requiring no isomerization along the dissociation coordinate. Relative energies at the B3LYP/aug-cc-pVDZ level in $\mathrm{kJ} / \mathrm{mol}$. 


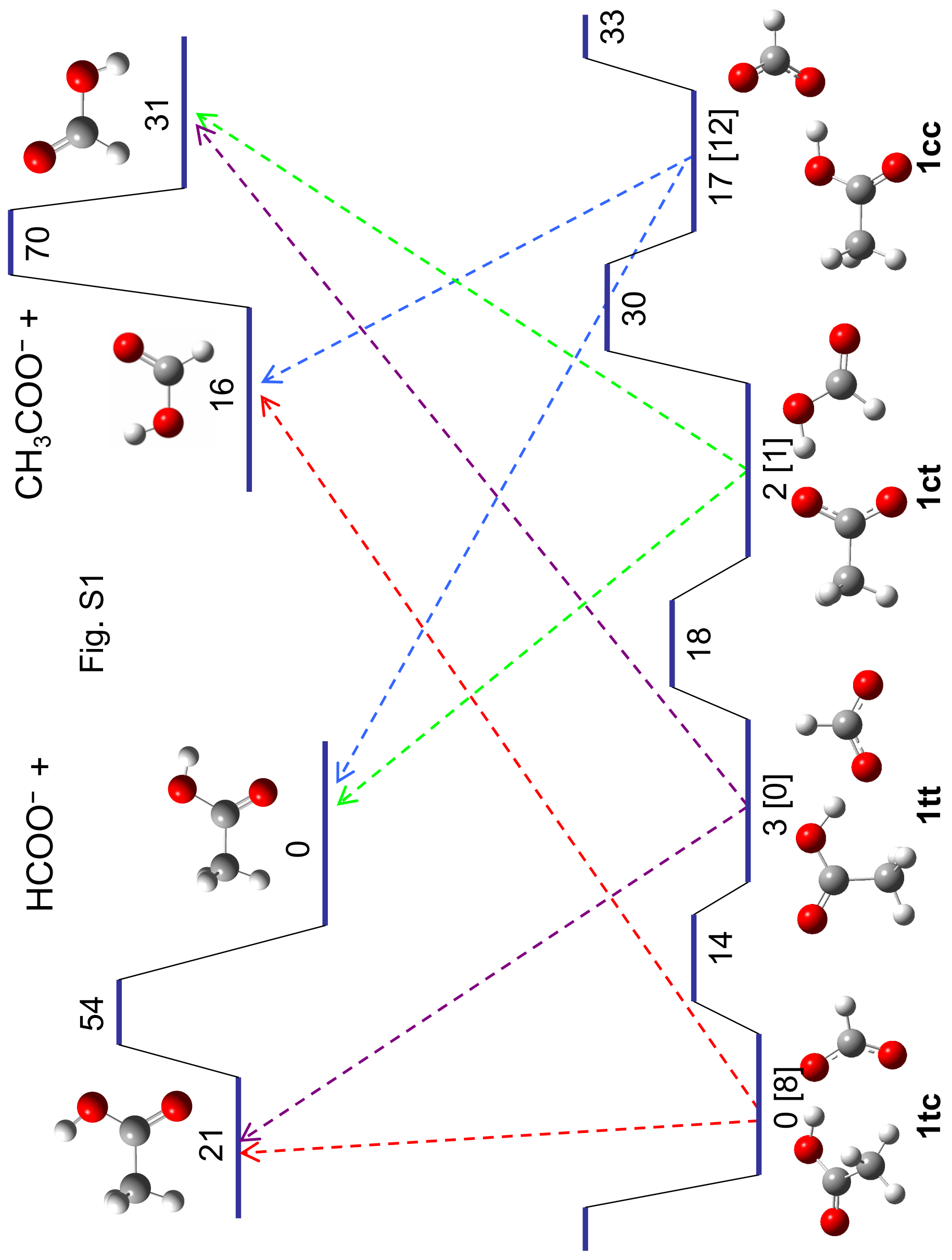




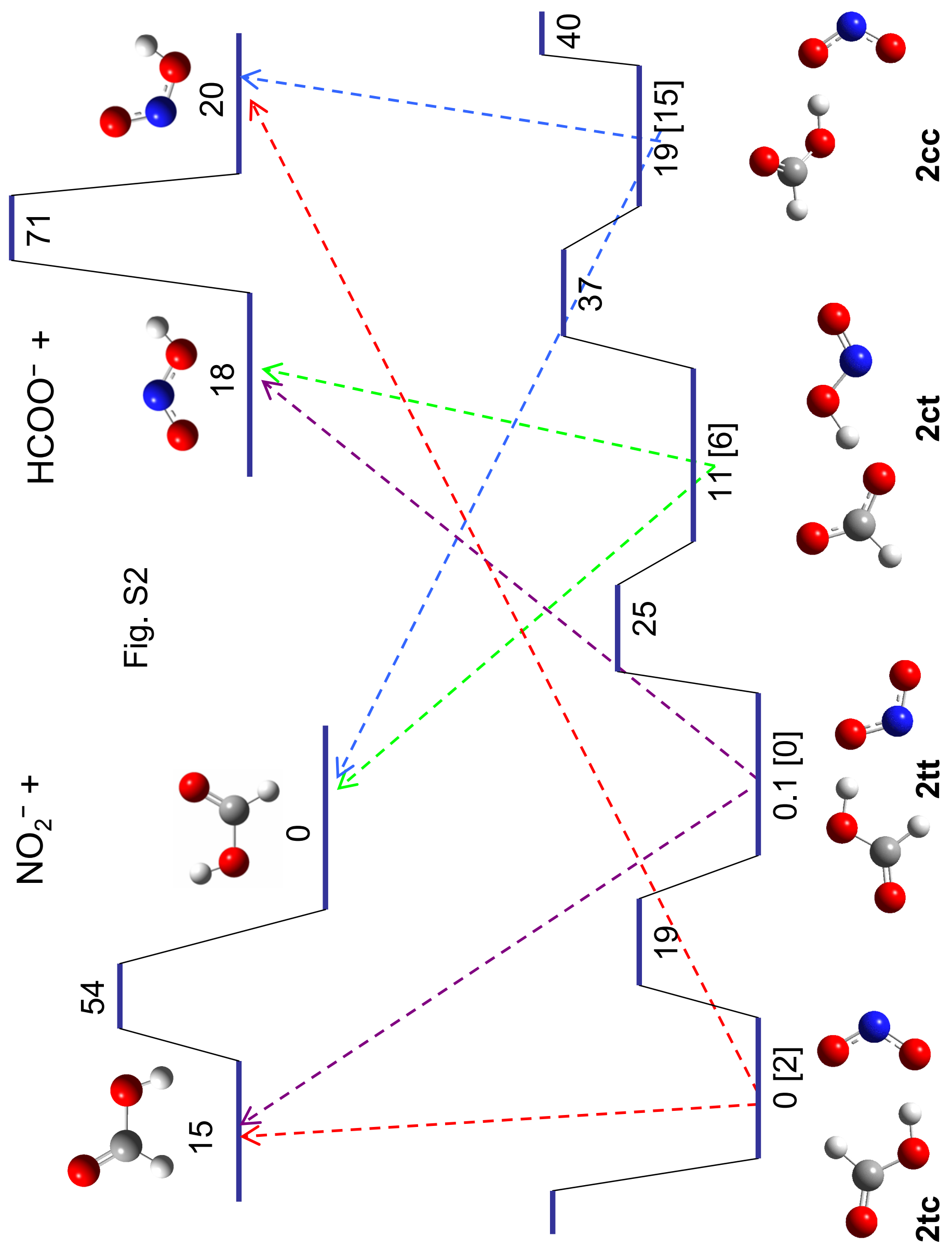




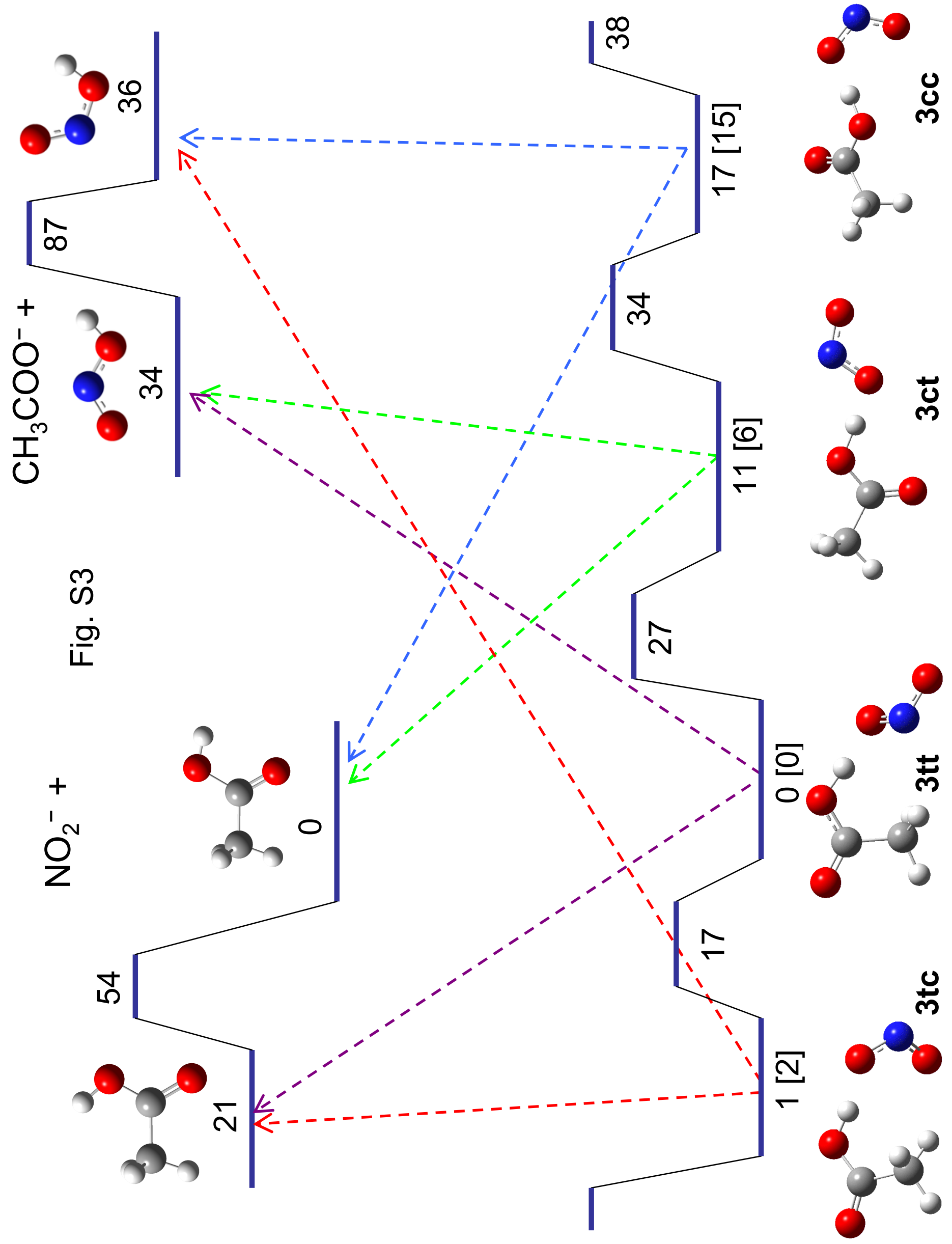




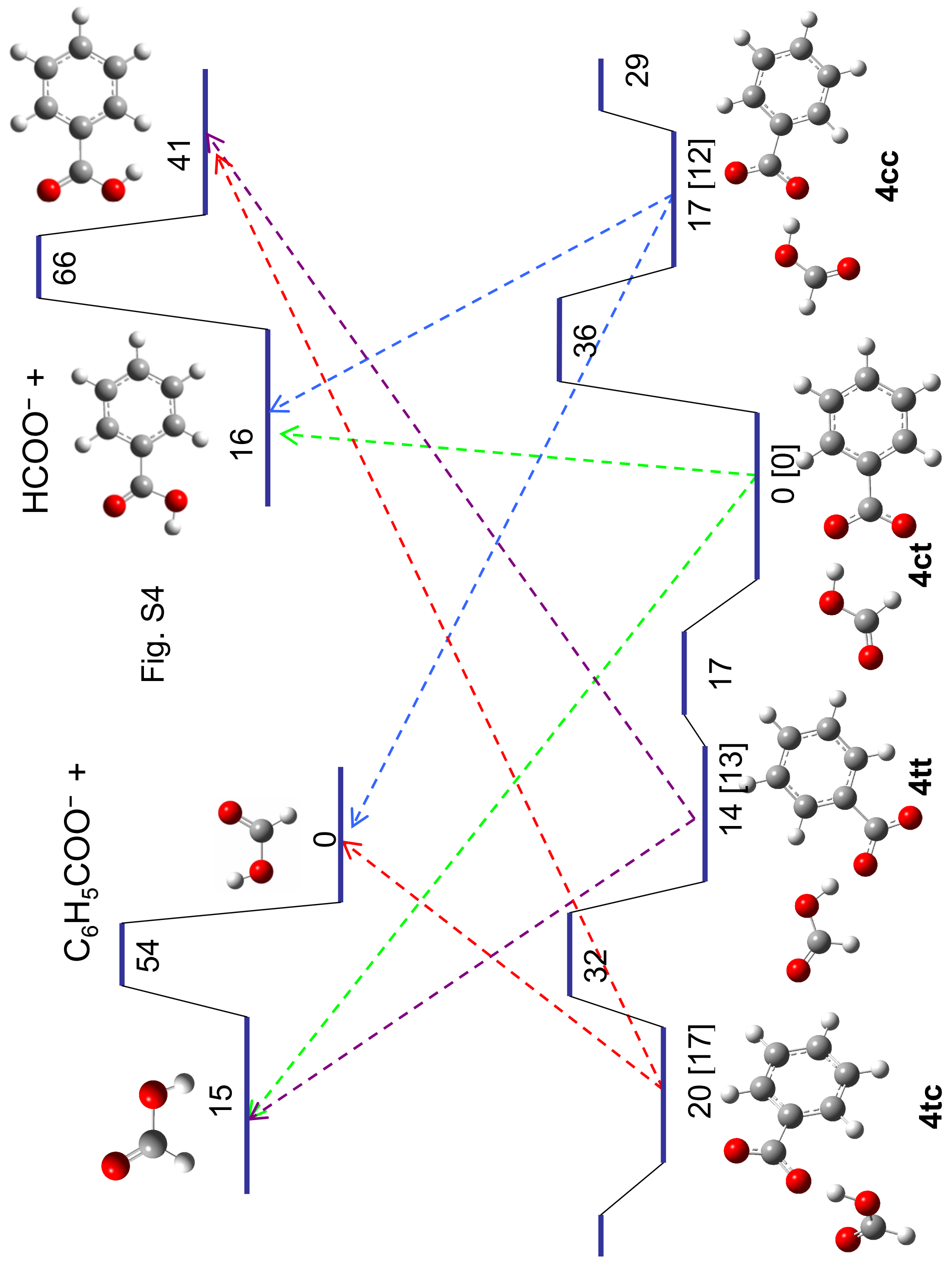




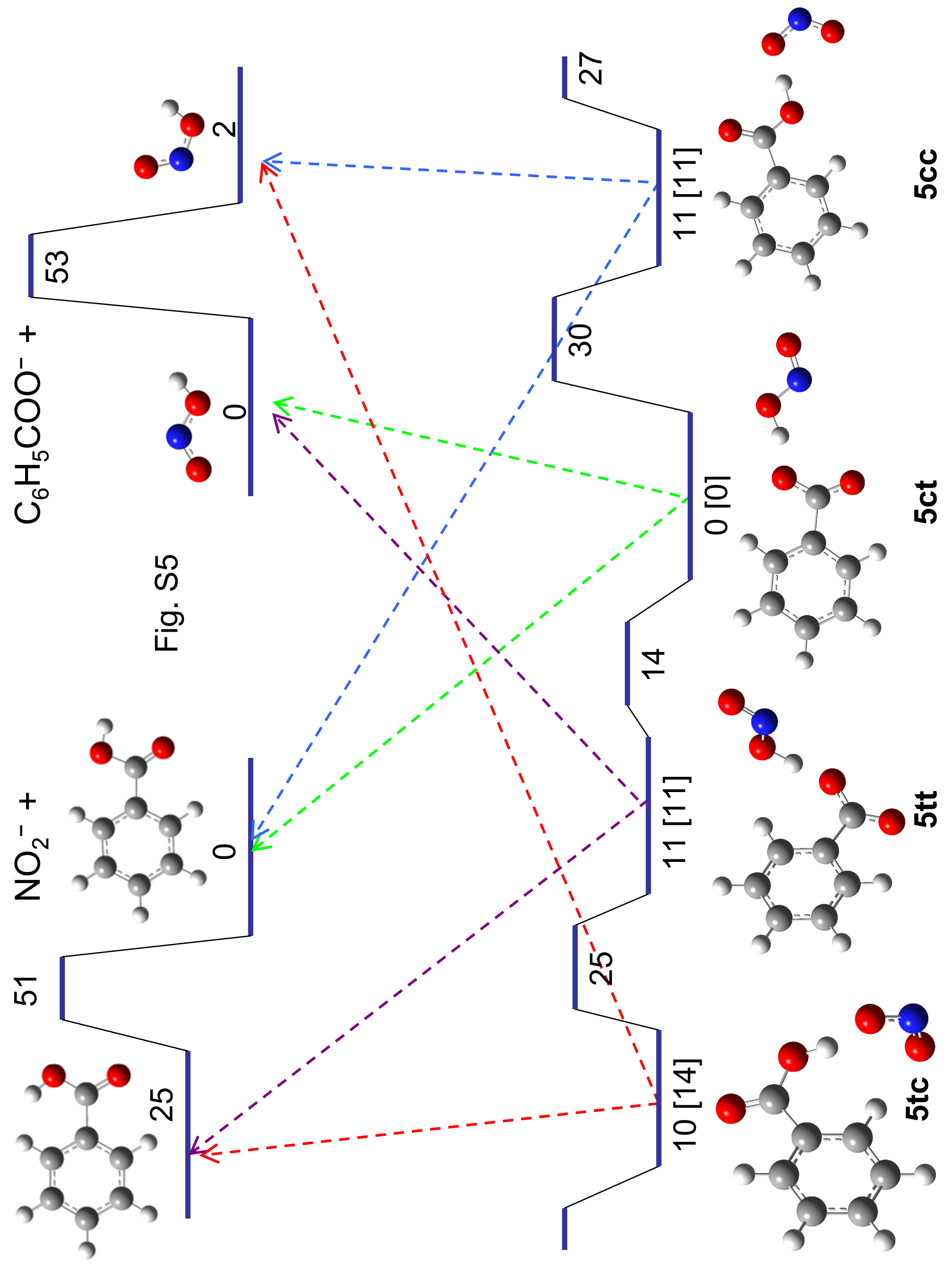

